\title{
Estudio Morfométrico del Seno Maxilar Mediante Tomografías Computadorizadas. Evaluación de la Reconstrucción Total de Piso Sinusal
}

\author{
Morphometric Study of Maxillary Sinus by Computed Tomography. \\ Assessment of Sinus Floor Bone Reconstruction \\ "Paulo Hemerson de Moraes; "Marcelo Victor Omena Caldas Costa; *Sergio Olate; \\ ****Paulo Henrique Ferreira Caria \& *José Ricardo de Albergaria Barbosa
}

DE MORAES, P. H.; COSTA, V. O. C.; OLATE, S.; CARIA, P. H. F. \& BARBOSA, J. R. A. Estudio morfométrico del seno maxilar mediante tomografías computadorizadas. Evaluación de la reconstrucción total de piso sinusal. Int. J. Morphol., 30(2):592-598, 2012.

RESUMEN: La rehabilitación con implantes dentales en maxila posterior es compleja debido a la presencia del seno maxilar con sus diferentes variaciones anatómicas; el objetivo de este trabajo fue determinar el volumen óseo intrasinusal necesario para realizar la instalación de uno o mas implantes dentarios sin invadir la membrana sinusal. En 22 cráneos fueron evaluados 40 senos maxilares mediante tomografías computadorizadas (TC) realizando mediciones en los cortes axiales, coronales y sagitales. Se clasificaron los senos maxilares según el remanente de hueso alveolar y se instalo un implante de forma virtual en el lugar del primer molar superior, mediante el software implant view. Con estas medidas volumétricas finales se estableció las indicaciones reconstructivas intra sinusales a partir de los sitios donantes intrabucales mas frecuentemente evaluados en la literatura mundial. El grupo I ( $1 \mathrm{~mm}$ a $4 \mathrm{~mm}$ de reborde alveolar remanente) presentaba déficit óseo de hasta 1,98cm3, el grupo II (4mm a $7 \mathrm{~mm}$ ) presentaba déficit de 1,06 $\mathrm{cm} 3$; el grupo III $(7 \mathrm{~mm}$ a $10 \mathrm{~mm})$ presentaba un déficit de $0,67 \mathrm{~cm} 3$; a partir de estos resultados podemos concluir que los sitios intrabucales pueden ser utilizados en la mayoría de los grupo estudiados, incluyendo las reconstrucciones bilaterales.

PALABRAS CLAVE: Seno maxilar; Elevación de seno maxilar; Reconstrucción ósea.

\section{INTRODUCCIÓN}

En maxila posterior edéntula existen limitaciones anatómicas para la instalación de implantes oseointegrados, destacando la deficiencia ósea en términos del volumen del hueso alveolar remanente y la presencia del seno maxilar, de mayor volumen en pacientes edéntulos; en estos casos, la alternativa de rehabilitación se asocia a la instalación de implantes con diseños modificados, lo cual presenta algunas limitaciones (Olate et al. 2010) y la reconstrucción ósea parcial de forma previa a la instalación de implantes (Raghoebar et al. 1997).

En 1980, Boyne \& James, realizaron una de las primeras publicaciones abordando la cirugía destinada al trasplante óseo a través de una ostectomia realizada en la pared lateral del seno maxilar; el injerto óseo en forma de partículas era instalado dentro del seno maxilar, posterior al descolamiento de la mucosa sinusal, lo que permitía la integración ósea intrasinusal.
El hueso autógeno es el patrón de oro en la reconstrucción ósea, que, aplicado antes o durante la instalación de implantes oseointegrados, optimiza los tratamiento y mejora los resultados estéticos y funcionales (Olate et al. 2007a), siendo el único material capaz de entregar las propiedades osteoconductivas, osteoinductivas y osteogénicas (Becker et al., 1995, Johansson et al., 2010). De esta forma, la obtención de estos injertos se realiza a partir de la sínfisis mandibular, rama mandibular, proceso coronoide, hueso palatino, hueso cigomático, parietal, epífisis de tibia y hueso de cresta iliaca, entre otras (Uchida et al., 1998, Hassani et al., 2005, Rocha et al., 2010,).

En los injertos menores, que generalmente no abarcan más de 2 o 3 implantes, los sitios intraorales presentan una gran versatilidad, incorporando rapidez, precisión, disminución de la morbilidad y bajo tiempo quirúrgico (Olate et al., 2007b); los procedimientos son de tipo ambulatorio,

\footnotetext{
" Área de Cirugía Oral y Maxilofacial, Depto. de Diagnóstico Oral, Facultad de Odontologia de Piracicaba, Universidad Estadual de Campinas, Brasil.

** División de Cirugía Oral y Maxilofacial, Depto. de Odontología Integral, Facultad de Medicina, Universidad de La Frontera, Chile.

*** Área de Anatomia, Depto. de Morfologia, Facultad de Odontología de Piracicaba, Universidad Estadual de Campinas, Brasil.
} 
sin hospitalización y generalmente con la única aplicación de anestésico local, lo cual disminuye los costos económicos del procedimiento (Olate et al., 2007b); por otra parte, la capacidad de regeneración ósea es observada en estos injertos, situación no observada en buena parte de los materiales no autógenos (Chaves Netto et al., 2009). Finalmente, la principal desventaja del sitio intraoral está asociada a la menor cantidad de hueso que puede ser recolectado (Jensen et al., 1994, Hirsch et al.,1991).

Por esta razón, es fundamental conocer la deficiencia ósea del lugar a implantar, que en el caso del seno maxilar involucra el volumen óseo necesario para reconstruir parcialmente la altura y el ancho óseo necesario, determinado por la cantidad y características del implante que se desea instalar. El saber anticipadamente el tipo de defecto óseo, permite calcular la cantidad de hueso que será necesario retirar (Hallman et al., 2002). Conociendo la cantidad necesaria de hueso, se puede escoger con mayor precisión el sitio donante y junto a ello, las técnicas anestésicas y el tiempo quirúrgico aproximado (Krenmair et al., 2006).

El objetivo de esta investigación es determinar el volumen óseo necesario para instalar un implante de $11,5 \mathrm{~mm}$ de largo y $4,1 \mathrm{~mm}$ de diámetro ubicado en maxila posterior en maxilas edéntulas y parcialmente edéntulas.
Fueron analizados 22 cráneos humanos sin distinción de género o raza, con una edad estimada de 20 a 60 años; los cráneos debían presentar ausencia del primer molar superior junto a un alvéolo completamente reparado. De esta forma, fueron analizados 40 senos maxilares siendo ello 18 del lado derecho y 22 del lado izquierdo.

Se realizaron tomografías computadorizadas con cortes seriados axiales y coronales $(1 \mathrm{~mm})$, a partir del cual se realizo la reconstrucción para obtención de la imagen sagital; posteriormente se utilizo el software Digital Imaging and Communications in Medicine (DICOM ${ }^{\circledR}$ ) para realizar las reconstrucciones tridimensionales; después de esta etapa, fue utilizado el software Implant Viewer 2 (Anne Solutions $\left.{ }^{\circledR}\right)$ para realizar la instalación virtual de implantes de $4,3 \mathrm{~mm}$ de diámetro y $11,5 \mathrm{~mm}$ de altura. Un observador fue responsable de realizar las mediciones y los procedimientos computacionales (Fig. 1).

Inicialmente fueron realizadas las mediciones de la altura ósea inferior al seno maxilar en la región del primer molar; cada seno maxilar fue incluido dentro de tres grupos diferentes, donde el grupo I presentaba de $1 \mathrm{~mm}$ a $4 \mathrm{~mm}$ de altura ósea alveolar remaneciente, el grupo II de $4 \mathrm{~mm}$ a $7 \mathrm{~mm}$ de altura ósea alveolar remanente y el grupo III con $7 \mathrm{~mm}$ a $10 \mathrm{~mm}$ de altura ósea.

\section{MATERIAL Y MÉTODO}

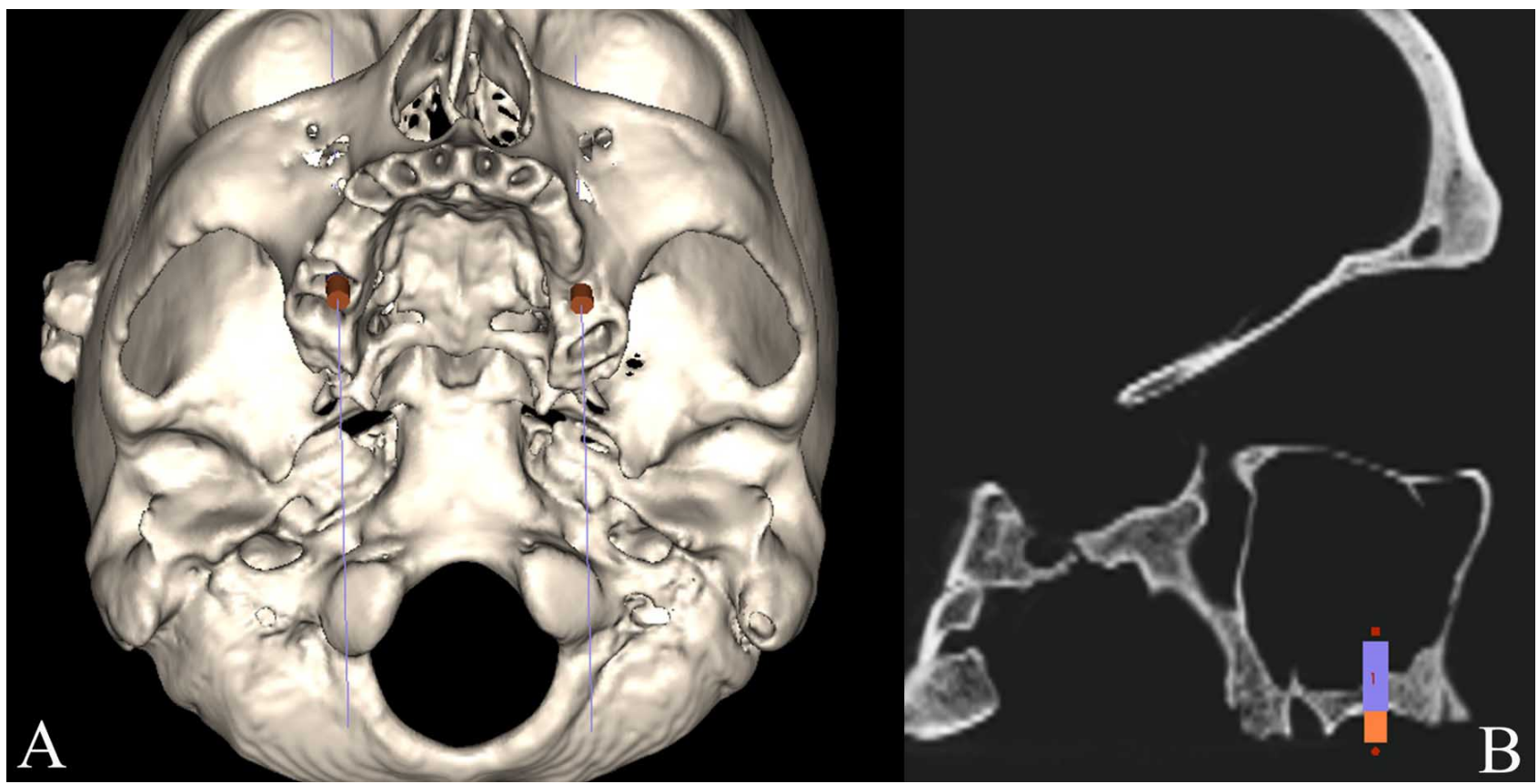

Fig. 1. (A) Vision del craneo reconstruido virtualmente con la instalación de implantes de 11,5 mm de largo en region del primer molar. (B) Imagen sagital con visualización del implante instalado con $6 \mathrm{~mm}$ de invación en el seno maxilar. 


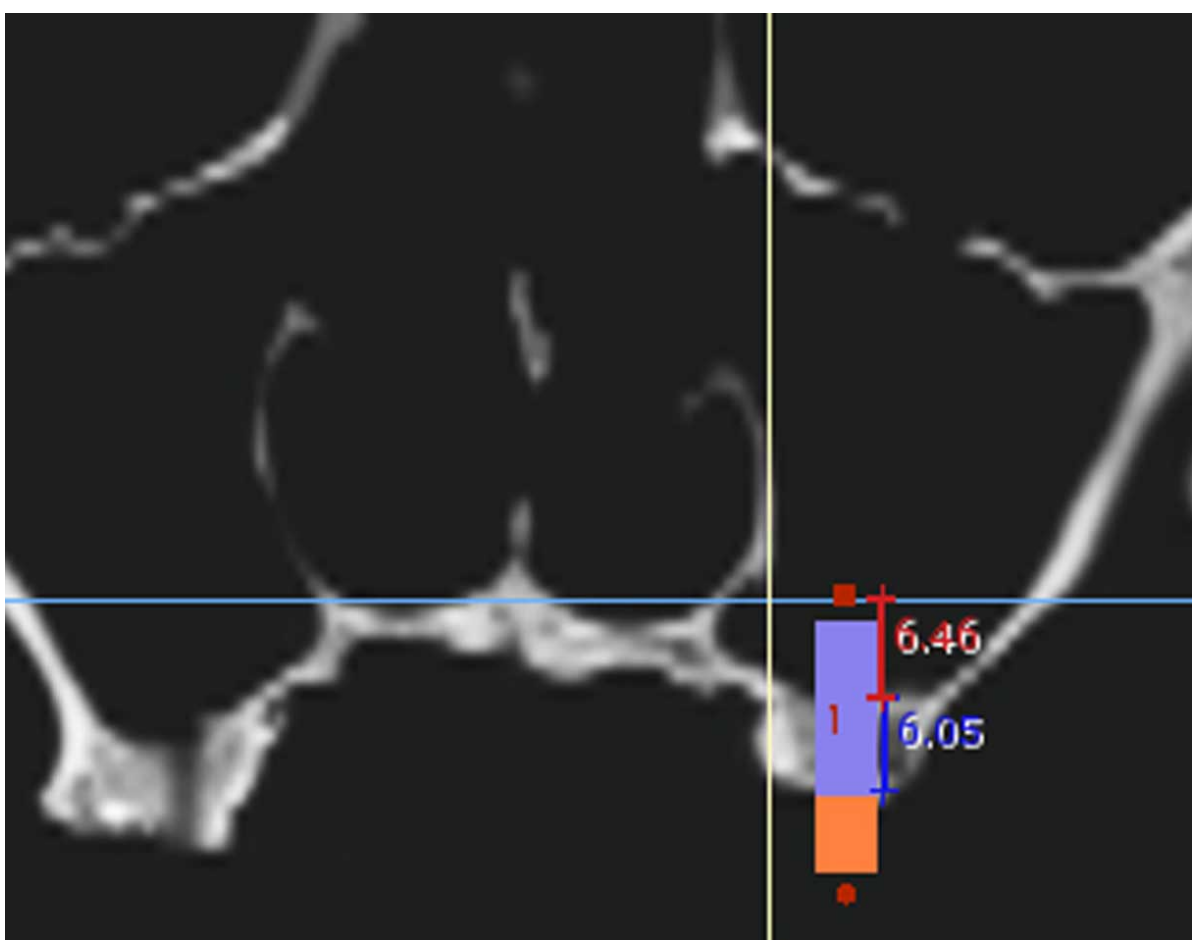

Fig. 2. Visión coronal del seno maxilar donde se observa la instalación del implante en un área de deficiencia de $6 \mathrm{~mm}$ de altura, señalando la necesidad de reconstrucción ósea intrasinusal previa.

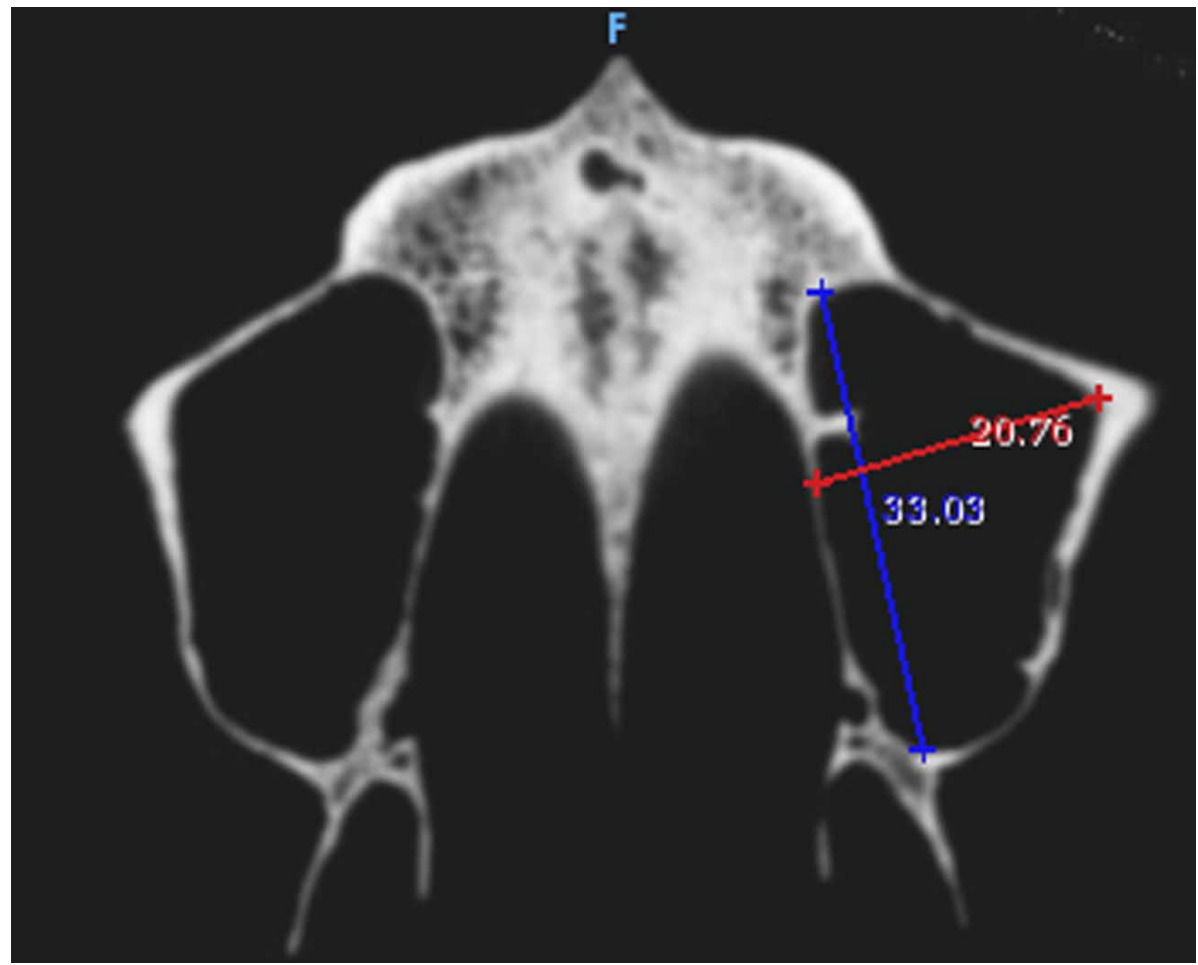

Fig. 3. Mediciones morfométricas antero posterior y medio lateral que señalan las dimensiones sinusales asociadas al proceso reconstructivo que debe ser realizado para la instalación de implante.
En la etapa de la instalación virtual del implante, se realizaron las mediciones adicionando un milímetro de profundidad a la instalación del mismo (Fig. 2); en la imagen axial se obtuvo las dimensiones antero-posteriores y medio lateral del seno maxilar (Fig. 3) y en la imagen coronal se obtuvo la altura ósea relacionada al implante instalado; a partir de estas situaciones fueron realizados los cálculos matemáticos para establecer la cantidad de tejido óseo necesario para cubrir completamente el implante (Fig. 4), obteniendo el volumen máximo que debería ser injertado para la instalación de uno o más implantes en base a una reconstrucción total de piso sinusal.

\section{RESULTADOS}

Los 40 senos maxilares analizados fueron distribuidos en el grupo I donde fueron incorporados 14 senos maxilares, en el grupo II con 16 senos y en el grupo III con 10 senos maxilares. Los valores por unidad de muestra están descritos en las Tablas I, II y III, en los sentidos sagitales, coronales y transversales.

El grupo I presentaba una deficiencia en altura ósea promedio de 9,97mm, señalando que prácticamente no existía remanente óseo para la instalación e implantes; en el caso del volumen de injerto óseo total necesario para este grupo se determino que $1,96 \mathrm{~cm}^{3}$ sería necesario para reconstruir el déficit óseo.

Para el grupo II, la deficiencia ósea en altura presento una media de $6,53 \mathrm{~mm}$ con un volumen óseo necesario para injer- 


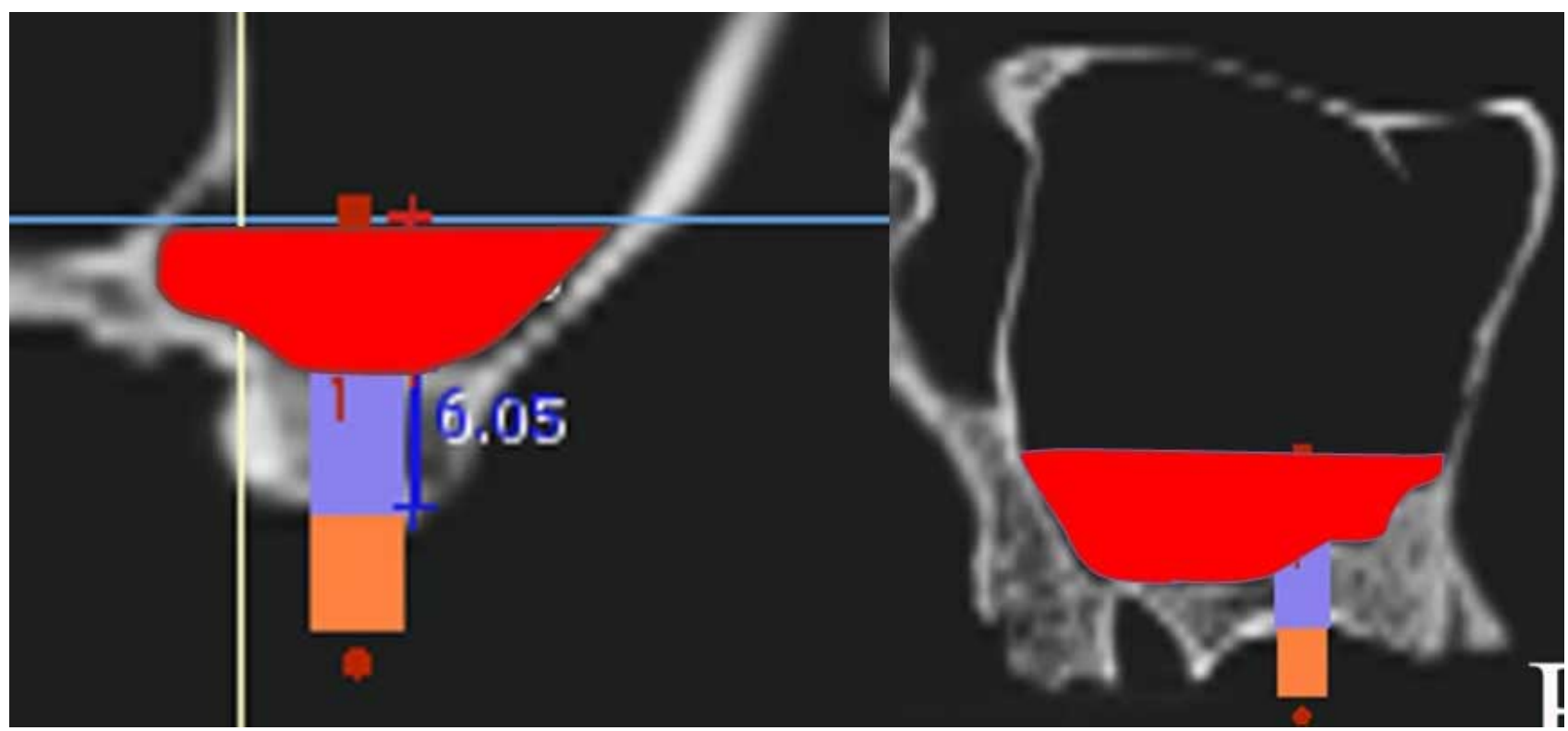

Fig. 4. El volumen óseo necesario para injertar se obtuvo del producto de las mediciones tridimensionales señalando la totalidad del área a ser injertada. Indudablemente, toda el área sinusal señalada como para ser injertada, no es necesaria para instalar solo un implante, pero si lo puede llegar a ser cuando se piensa en la instalación de 3 implantes.

tar de $1,1 \mathrm{~cm}^{3}$, casi $1 \mathrm{~cm}^{3}$ menos que en el grupo I. En el grupo III, la deficiencia de altura ósea fue de $4,05 \mathrm{~mm}$ con un volumen óseo a injertar de $0,44 \mathrm{~cm}^{3}$.

La morfometría sagital (anteroposterior) también presento variaciones según los grupos, siendo de $17,84 \mathrm{~mm}$ en el grupo I; $15,49 \mathrm{~mm}$ en el grupo II y $14,72 \mathrm{~mm}$ en el grupo III. La morfometría transversal del seno maxilar fue de $32,24 \mathrm{~mm}$ en el grupo I; $27,94 \mathrm{~mm}$ en el grupo II y $29,28 \mathrm{~mm}$ en el grupo III.

Tabla I. Características morfométricas de 14 senos maxilares con altura de reborde alveolar de entre $1 \mathrm{~mm}$ y $3 \mathrm{~mm}$

\begin{tabular}{|c|c|c|c|c|c|c|c|c|}
\hline $\begin{array}{c}\text { Seno } \\
\text { maxilar }\end{array}$ & $\begin{array}{l}\text { Altura } \\
\text { R. A. }\end{array}$ & DE & $\begin{array}{l}\text { Altura necesaria del } \\
\text { injerto (implante de } \\
\qquad 11,5 \mathrm{~mm})\end{array}$ & $\begin{array}{c}\text { Distancia } \\
\text { Sagital }\end{array}$ & DE & $\begin{array}{c}\text { Distancia } \\
\text { transversal }\end{array}$ & DE & $\begin{array}{c}\text { Volumen } \\
\text { sinusal }\end{array}$ \\
\hline 1 & 1,2 & 0,21 & 11,3 & 23,09 & 0,30 & 36,01 & 0,33 & 3131,9 \\
\hline 2 & 1,4 & 0,20 & 11,1 & 17,04 & 0,36 & 33,45 & 0,34 & 2109 \\
\hline 3 & 1,5 & 0,16 & 11 & 18,71 & 0,31 & 37,14 & 0,32 & 2547,9 \\
\hline 4 & 1,7 & 0,15 & 10,8 & 16,84 & 0,28 & 33,46 & 0,30 & 2028,5 \\
\hline 5 & 1,87 & 0,13 & 10,63 & 16,66 & 0,29 & 32,28 & 0,29 & 1905,6 \\
\hline 6 & 2,02 & 0,20 & 10,48 & 16,14 & 0,33 & 19,89 & 0,35 & 1682,2 \\
\hline 7 & 2,09 & 0,18 & 10,41 & 14,77 & 0,32 & 32,21 & 0,36 & 1650,8 \\
\hline 8 & 2,33 & 0,14 & 10,17 & 18,42 & 0,34 & 32,52 & 0,37 & 2030,7 \\
\hline 9 & 2,56 & 0,12 & 9,94 & 17,74 & 0,27 & 35 & 0,24 & 2057,2 \\
\hline 10 & 3,41 & 0,21 & 9,09 & 16,88 & 0,21 & 36,46 & 0,23 & 1864,8 \\
\hline 11 & 3,49 & 0,13 & 9,01 & 17,58 & 0,32 & 31,95 & 0,30 & 1686,9 \\
\hline 12 & 3,95 & 0,18 & 8,55 & 17,45 & 0,29 & 30,98 & 0,24 & 1540,7 \\
\hline 13 & 3,95 & 0,16 & 8,55 & 22,59 & 0,32 & 24,34 & 0,30 & 1567 \\
\hline 14 & 3,96 & 0,12 & 8,54 & 15,81 & 0,37 & 35,64 & 0,32 & 1604 \\
\hline Total & 2,53 & 0,16 & 9,97 & 17,84 & 0,31 & 32,24 & 0,31 & 1957,66 \\
\hline
\end{tabular}

$\mathrm{DE}=$ Desviación estándar, $\mathrm{RA}=$ Reborde alveolar, Valores en mm y en mm3. 
Tabla II. Características morfométricas de 16 senos maxilares con altura de reborde alveolar de entre $4 \mathrm{~mm}$ y $7 \mathrm{~mm}$

\begin{tabular}{|c|c|c|c|c|c|c|c|c|}
\hline $\begin{array}{c}\text { Seno } \\
\text { Maxilar }\end{array}$ & $\begin{array}{l}\text { Altura } \\
\text { R. A. }\end{array}$ & $\mathrm{DE}$ & $\begin{array}{l}\text { Altura necesaria del } \\
\text { injerto (Implante de } \\
\qquad 11,5 \mathrm{~mm})\end{array}$ & $\begin{array}{c}\text { Distancia } \\
\text { anteroposterior }\end{array}$ & $\mathrm{DE}$ & $\begin{array}{c}\text { Distancia } \\
\text { medio lateral }\end{array}$ & $\mathrm{DE}$ & $\begin{array}{c}\text { Volumen } \\
\text { sinusal }\end{array}$ \\
\hline 1 & 4,42 & 0,25 & 8,08 & 14,75 & 0,45 & 25,35 & 0,42 & 1007,1 \\
\hline 2 & 4,89 & 0,22 & 7,61 & 16,1 & 0,47 & 37 & 0,43 & 1511,1 \\
\hline 3 & 5,12 & 0,26 & 7,38 & 12,95 & 0,44 & 29,07 & 0,45 & 926,08 \\
\hline 4 & 5,12 & 0,23 & 7,38 & 8,89 & 0,43 & 26,72 & 0,42 & 584,35 \\
\hline 5 & 5,36 & 0,24 & 7,14 & 13,92 & 0,45 & 13,53 & 0,46 & 448,24 \\
\hline 6 & 5,67 & 0,25 & 6,83 & 14,68 & 0,43 & 32,23 & 0,44 & 1077,2 \\
\hline 7 & 5,95 & 0,27 & 6,55 & 15,6 & 0,46 & 30,75 & 0,44 & 1047,3 \\
\hline 8 & 6,05 & 0,24 & 6,51 & 17,56 & 0,47 & 30,22 & 0,45 & 1151,5 \\
\hline 9 & 6,21 & 0,23 & 6,29 & 25,33 & 0,43 & 17,41 & 0,47 & 924,62 \\
\hline 10 & 6,28 & 0,24 & 6,22 & 14,03 & 0,46 & 23,26 & 0,43 & 676,61 \\
\hline 11 & 6,28 & 0,26 & 6,22 & 16,95 & 0,43 & 33,97 & 0,46 & 1193,8 \\
\hline 12 & 6,6 & 0,27 & 5,9 & 14,39 & 0,46 & 25,04 & 0,44 & 708,64 \\
\hline 13 & 6,74 & 0,24 & 5,76 & 13,94 & 0,43 & 28,39 & 0,44 & 759,85 \\
\hline 14 & 6,95 & 0,24 & 5,55 & 16,15 & 0,45 & 34,42 & 0,46 & 1028,4 \\
\hline 15 & 6,98 & 0,27 & 5,52 & 12,69 & 0,44 & 25,52 & 0,43 & 595,88 \\
\hline 16 & 6,98 & 0,25 & 5,52 & 19,87 & 0,48 & 34,22 & 0,41 & 1251,1 \\
\hline Total & 5,96 & 0,25 & 6,53 & 15,49 & 0,44 & 27,94 & 0,44 & 930,73 \\
\hline
\end{tabular}

Tabla III. Características morfométricas de 10 senos maxilares con altura de reborde alveolar de entre $7 \mathrm{~mm}$ y $10 \mathrm{~mm}$

\begin{tabular}{ccccccccc}
\hline $\begin{array}{c}\text { Seno } \\
\text { maxilar }\end{array}$ & $\begin{array}{c}\text { Altura } \\
\text { R. A. }\end{array}$ & DE & $\begin{array}{c}\text { Altura necesaria del } \\
\text { injerto (Implante de } \\
11,5 \mathrm{~mm})\end{array}$ & $\begin{array}{c}\text { Distancia } \\
\text { anteroposterior }\end{array}$ & DE & $\begin{array}{c}\text { Distancia } \\
\text { medio lateral }\end{array}$ & $\begin{array}{c}\text { DE } \\
\text { Volumen } \\
\text { sinusal }\end{array}$ \\
\hline 1 & 7,21 & 0,35 & 5,39 & 27,02 & 0,54 & 32,5 & 0,60 & 1577,7 \\
2 & 7,44 & 0,33 & 5,06 & 19 & 0,52 & 32,81 & 0,58 & 1051,5 \\
3 & 7,44 & 0,34 & 5,06 & 10,56 & 0,57 & 25,45 & 0,55 & 453,3 \\
4 & 8,14 & 0,37 & 4,36 & 11,69 & 0,53 & 23,38 & 0,58 & 397,21 \\
5 & 8,21 & 0,36 & 4,29 & 13,25 & 0,51 & 30,79 & 0,59 & 583,39 \\
6 & 8,54 & 0,35 & 3,96 & 13,13 & 0,56 & 26,26 & 0,60 & 455,13 \\
7 & 9,3 & 0,35 & 3,2 & 8,76 & 0,54 & 26,28 & 0,45 & 245,56 \\
8 & 9,3 & 0,34 & 3,2 & 13,24 & 0,54 & 32,52 & 0,48 & 459,27 \\
9 & 9,52 & 0,35 & 2,98 & 12,1 & 0,53 & 31,42 & 0,59 & 377,65 \\
10 & 9,53 & 0,36 & 2,97 & 18,42 & 0,52 & 31,37 & 0,50 & 572,06 \\
\hline Total & $\mathbf{8 , 4 6}$ & $\mathbf{0 , 3 5}$ & $\mathbf{4 , 0 5}$ & $\mathbf{1 4 , 7 2}$ & $\mathbf{0 , 5 7}$ & $\mathbf{2 9 , 2 8}$ & $\mathbf{0 , 5 5}$ & $\mathbf{6 1 7 , 2 8}$ \\
\hline
\end{tabular}

$\mathrm{DE}=$ Desviación estándar, $\mathrm{RA}=$ Reborde alveolar, Valores en $\mathrm{mm}$

\section{DISCUSIÓN}

El seno maxilar ha sido evaluado por diferentes autores y se ha demostrado el éxito en los tratamientos con reconstrucción ósea (Chiapasco \& Zaniboni 2009). Chiapasco et al. (2008) desarrollaron una clasificación de los senos maxilares basadas en las necesidades rehabilitadoras, las condiciones anatómicas del seno maxilar y las técnicas quirúrgicas aplicadas, señalando 9 divisionesdonde el remanente alveolar era fundamental para establecer los procedimientos; nuestra investigación propone la simple división en tres grupos basados, al igual que Chiapasco et al., en el remanente alveolar y a partir de ello la necesidad volumétrica de la reconstrucción ósea; esta clasificación responde a la necesidad de reconocer la necesidad de volumen óseo para la reconstrucción intra sinusal y puede ser util para la planificación o para la técnica quirúrgica como lo propone Chiapasco et al. 
Para los diferentes grupos, la necesidad total de reconstrucción fue próximo a los $2 \mathrm{~cm}^{3}$ (grupo I), $1 \mathrm{~cm}^{3}$ (grupo II) y $0,6 \mathrm{~cm}^{3}$ (grupo III).

De la rama mandibular se pueden obtener injertos óseos de dimensiones próximas a los 4,0mm de espesura, pudiendo llegar a $35 \mathrm{~mm}$ de largo y $15 \mathrm{~mm}$ de ancho (sentido sagital), lo que entrega un volumen probable de $2,1 \mathrm{~cm}^{3}$, aplicable en cualquiera de los grupos estudiados (Misch et al., 1996). Li \& Schwartz (1996) determinaron que era posible obtener injerto de cuerpo mandibular próximos a los $13 \mathrm{~mm}$ de largo y $3 \mathrm{~mm}$ de ancho, posibilitando su uso también en estos grupos. Montazem et al. (2000) evaluaron el volumen óseo que puede ser obtenido de sínfisis mandibular, respetando los límites que impidan lesión dentaria o nerviosa (nervio mental) en 16 cadáveres, estableciendo un promedio de $4,84 \mathrm{~cm} 3$, lo cual implica que puede ser empleado en los casos de mayor aumento óseo y en casos de reconstrucción osea intra sinusal bilateral.
El hueso zigomático fue estudiado por Kainulainen et al. (2004), donde analizaron 20 cráneos y 40 áreas zigomáticas, observando que el hueso de este sitio, aplicado en forma de partículas, era posible manejar cerca de $0,53 \mathrm{~mm}^{3}$, lo cual limita su aplicación a los defectos menores presentes en el grupo III. Chuong \& Kim en el 2000 presentaron una investigación donde señalaron también la opción de tratar los defectos señalados con injerto obtenido del proceso coronoide, pues esta estructura presentarían volúmenes próximos a los $0,9 \mathrm{~cm} 3$, lo cual permitiría su aplicación a casos del grupo II y III.

En esta investigación no fueron evaluados los procedimientos reconstructivos asociados a injertos en bloque extra sinusal; sin embargo podemos concluir que para los senos maxilares evaluados con TC, los sitios donantes intrabucales pueden ser utilizados para la elevación de piso de seno maxilar, incluyendo los casos simples, casos complejos e incluso casos de tratamiento bilateral.

DE MORAES, P. H.; COSTA, V. O. C.; OLATE, S.; CARIA, P. H. F. \& BARBOSA, J. R. A. Morphometric study of maxillary sinus by computed tomography. Assessment of sinus floor bone reconstruction. Int. J. Morphol., 30(2):592-598, 2012.

SUMMARY: Rehabilitation with dental implants in posterior maxilla is difficult because of the presence of maxillary sinus with anatomical variations. The aim of this research was to evaluate the sinus volume to install one or more implants without invading the sinus membrane. Forty (40) maxillary sinuses were evaluated in 22 skulls by computed tomography measuring in the axial, coronal and sagittal slice. The sinus were classified according to the remaining alveolar bone and a dental implant in the place of the upper first molar was virtually installed by implant view software. With this final volumetric measurement the surgical indications were established for inlay reconstruction from intra oral donor sites frequently evaluated in world literature. Group I ( $1 \mathrm{~mm}$ to $4 \mathrm{~mm}$ of alveolar ridge) showed a bone deficit of $1.98 \mathrm{~cm}^{3}$, group II $(4 \mathrm{~mm}$ to $7 \mathrm{~mm})$ showed a deficit of $1.06 \mathrm{~cm}^{3}$; group III ( $7 \mathrm{~mm}$ to $\left.10 \mathrm{~mm}\right)$ showed a $0.67 \mathrm{~cm}^{3}$ deficit. In view of these results we concluded that intra oral donor site can be used for the three study groups and in some cases in bilateral reconstruction.

KEY WORDS: Maxillary sinus; Sinus lift; Bone reconstruction.

\section{REFERENCIAS}

Becker, W.; Schenk, R.; Higuchi, K.; Lekholm, U.\& Becker, B. E. Variations in bone regeneration adjacent to implants augmented with barrier membranes alone or with demineralized freeze-dried bone or autologous grafts: $a$ study in dogs. Int. J. Oral Maxillofac. Implants 10:143-54, 1995.

Boyne, P.; J. \& James, R. A. Grafting of the maxillary sinus floor with autogenous marrow and bone. J. Oral Surg 38:613-6, 1980.

Chaves Netto, H. D. M.; Olate, S.; Miranda Chaves, M. G. A.; Albergaria-Barbosa, J. R. \& Mazzonetto, R. Análisis histológico del proceso de reparación em defectos óseos. Reconocimiento de defectos críticos. Int. J. Morphol., 27:11217, 2009.
Chiapasco, M.; Zaniboni, M. \& Rimondini, L. Dental implants placed in grafted maxillary sinus: a retrospective analysis of clinical outcome according to the initial clinical situation and a proposal of defect classification. Clin. Oral Implants Res. 19:416-28, 2008.

Chiapasco, M. \& Zanibone, M. Methods to treat the edentulous posterior maxilla: implants with sinus grafting. J. Oral Maxillofac. Surg., 67:867-71, 2009.

Chuong, P., H. \& Kim, S., G. The coronoid process for paranasal augmentation in the correction of midfacial concavity. Oral Surg. Oral Med. Oral Pathol., 91:28-33, 2001.

Hallman, M.; Sennerby, L. \& Lundgren, S. A clinical and histologic evaluation of implant integration in the posterior maxilla after 
sinus floor augmentation with autogenous bone, bovine hydroxyapatite or a 20:80 mixture. Int. J. Oral Maxillofac. Implants 17:635-43, 2002.

Hassani, A.; Khojasteh, A. \& Shamsabad, A. N. The anterior palate as a donor site in maxillofacial bone grafting: a quantitative anatomic study. J. Oral Maxillofac. Surg., 63: 1196-200, 2005.

Hirsch, J. M. \& Ericsson, I. Maxillary sinus augmentation using mandibular bone grafts and simultaneous installation of implants. A surgical technique. Clin. Oral Impl. Res., 2: 91-6, 1991.

Jensen, J.; Sindet-Pedersen, S. \& Oliver, A., J. Varying treatment strategies for reconstruction of maxillary atrophy with implants: results in 98 patients. J. Oral Maxillofac. Surg. 52:210-6, 1994.

Johansson, L. A.; Isaksson, S.; Lindh, C.; Becktor, J. P. \& Sennerby, L. Maxillary sinus floor augmentation and simultaneous implant placement using locally harvested autogenous bone chips and bone debris: A prospective clinical study. J. Oral Maxillofac. Surg., 68:837-44, 2010.

Kainulainen, V. T.; Sàndor, G. K., B.; Clokie, C. M. L.; Keller, A. M. \& Oikarinen, K., S. The zygomatic bone as a potential donor site for alveolar reconstruction - a quantitative anatomic cadaver study. Int. J. Oral Maxillofac. Surg., 33: 786-91, 2004.

Krennmair, G.; Krainhöfner, M.; Maier, H.; Weinländer, M. \& Piehslinger, E. Computerized tomography-assisted calculation of sinus augmentation volume. J. Oral Maxillofac. Implants 21:907-13, 2006.

Li, K. K. \& Schwartz, H., C. Mandibular body bone in facial plastic and reconstructive surgery. Laryngoscope 106:504-6, 1996.

Misch, C. M. Ridge augmentation using mandibular ramus bone grafts for the placement of dental implants: presentation of a technique. Pract. Periodontics Aesthet. Dent., 8:127-35, 1996.

Montazem, A.; Valauri, D. V.; Buchhinder, D. \& St-Hilaire, H. The Mandibular symphsis as a donor site in maxillofacial bone grafting: A quantitative anatomic study. Int. J. Oral Maxillofac. Implants, 58:1368-71, 2000.

Olate, S.; de Oliveira, G. R.; Jaimes, M. \& Albergaria-Barbosa, J. R.; Recuperación ósea en procedimientos de reconstrucción e instalación de implantes. Int. J. Morphol. 25:649-57, 2007.

Olate, S.; Kluppel, L. E.; Chaves Netto, H. D., M.; Stabile, G. A. V.; Mazzonetto, R. \& Albergaria-Barbosa, J., R. Sitios donantes mandibulares en implantologia - Una evaluación clínica. Int. J. Odontostomat., 1:121-7, 2007.

Olate, S.; Lyrio, M., C., N.; de Moraes, M.; Mazzonetto, R.; Moreira, R., W., F. Influence of diameter and lenght of implant on early dental implant failure. J. Oral Maxillofac. Surg., 68: 414-9, 2010.
Rocha, F., de Oliveira, G., R.; Olate, S. \& Albergaria-Barbosa, J., R. Consideraciones clínicas en la obtención de injertos óseos intraorales. Técnica quirúrgica y evaluación de las complicaciones. Av. Periodon. Implantol., 22:71-6, 2010.

Raghoebar, G. M.; Vissink, A.; Reintsema, H. \& Batenburg, R. H. $\mathrm{K}$. Bone grafting of the floor of the maxillary sinus for the placement of endosseous implants. Br. J. Oral Maxillofac. Surg., 35:119-25, 1997.

Uchida, Y.; Goto, M.; Katsuki, T. \& Soejima, Y. Measurement of maxillary sinus volume using computerized tomographic images. Int. J. Oral Maxillofac. Implants, 13:811-8, 1998.

\section{Dirección de correspondencia \\ Prof. Dr. Sergio Olate M. \\ División de Cirugía Oral \& Maxilofacial \\ Facultad de Medicina \\ Universidad de La Frontera \\ Claro Solar 115 \\ Temuco - CHILE}

Email: solate@ufro.cl

Recibido : 27-12-2011

Aceptado: 12-03-2012 